ARTICLE

DOI: $10.1038 / s 41467-018-03445-z$

OPEN

\title{
Micro-scale fusion in dense relativistic nanowire array plasmas
}

\author{
Alden Curtis ${ }^{1,3}$, Chase Calvi ${ }^{2}$, James Tinsley ${ }^{3}$, Reed Hollinger ${ }^{1}$, Vural Kaymak ${ }^{4}$, Alexander Pukhov ${ }^{4}$, \\ Shoujun Wang ${ }^{1}$, Alex Rockwood ${ }^{2}$, Yong Wang ${ }^{1}$, Vyacheslav N. Shlyaptsev ${ }^{1} \&$ Jorge J. Rocca,2 $^{1,2}$
}

Nuclear fusion is regularly created in spherical plasma compressions driven by multi-kilojoule pulses from the world's largest lasers. Here we demonstrate a dense fusion environment created by irradiating arrays of deuterated nanostructures with joule-level pulses from a compact ultrafast laser. The irradiation of ordered deuterated polyethylene nanowires arrays with femtosecond pulses of relativistic intensity creates ultra-high energy density plasmas in which deuterons (D) are accelerated up to MeV energies, efficiently driving D-D fusion reactions and ultrafast neutron bursts. We measure up to $2 \times 10^{6}$ fusion neutrons per joule, an increase of about 500 times with respect to flat solid targets, a record yield for joule-level lasers. Moreover, in accordance with simulation predictions, we observe a rapid increase in neutron yield with laser pulse energy. The results will impact nuclear science and high energy density research and can lead to bright ultrafast quasi-monoenergetic neutron point sources for imaging and materials studies.

\footnotetext{
${ }^{1}$ Department of Electrical and Computer Engineering, Colorado State University, Fort Collins, CO 80523, USA. ${ }^{2}$ Department of Physics, Colorado State University, Fort Collins, CO 80523, USA. ${ }^{3}$ Nevada National Security Site, Las Vegas, NV 89030, USA. ${ }^{4}$ Institut für Theoretische Physik, Heinrich-HeineUniversität Düsseldorf, 40225 Düsseldorf, Germany. Correspondence and requests for materials should be addressed to

J.J.R. (email: jorge.rocca@colostate.edu)
} 
S pherical compressions driven by multi-kilojoule lasers regularly produce fusion neutrons with typical yields on the order of $10^{4}-5 \times 10^{5}$ neutrons per joule $e^{1-3}$. Recent inertial confinement fusion (ICF) experiments at the National Ignition Facility used 1.9 megajoule laser pulses to produce a record $7.6 \times$ $10^{15}$ neutrons $\left(4 \times 10^{9}\right.$ neutrons per joule $)$ from deuterium-tritium fuel implosions ${ }^{4}$. In addition to ICF experiments, D-D fusion neutron bursts have been produced using energetic sub-ns pulses of a few hundred joules from chirpedpulse amplification lasers ${ }^{5,6}$, and using petawatt class lasers ${ }^{7-9}$. However, all these experiments are limited to repetition rates of a few shots per hour or less. The ability to drive fusion reactions with compact lasers that can be fired at much higher repetition rates is of significant interest for fusion science, high energy density studies, and neutron pulse generation. Specific applications of neutron sources include neutron imaging and tomography $^{10}$, neutron scattering ${ }^{11}$, and diffraction ${ }^{12}$ for the study of material structure and dynamics, and neutron and neutrino detector development. An early experiment with a compact femtosecond laser demonstrated the generation of fusion reactions producing 140 neutrons per shot from a deuterated polyethylene flat target irradiated at an intensity of $10^{18} \mathrm{~W} \mathrm{~cm}^{-213}$. Since then, several different fusion target geometries, target densities, and laser irradiation conditions have been investigated using compact lasers. The targets used include deuterated thin films ${ }^{6}$, cryogenic $\mathrm{D}_{2}{ }^{14}$, and deuterated clusters ${ }^{9,15-20}$. A significant advance in driving fusion reactions with compact lasers was the irradiation of deuterated clusters formed in gas jets with low energy femtosecond laser pulses, that allows for efficient volumetric heating of plasmas with an average ion density of $\sim 1 \times 10^{19} \mathrm{~cm}^{-315}$ in which cluster explosions accelerates ions to multi-keV average energy ${ }^{9}$. Neutron generation efficiencies of $\sim 1 \times 10^{5}$ neutrons per joule were obtained in the form of short sub-ns bursts, a value similar to those obtained with multikilojoule laser. The ultrafast irradiation of ordered nanowire arrays share with nanoclusters the advantage of efficient volumetric heating, but have the additional advantage of creating a media with several orders of magnitude higher average plasma density $^{21}$. We have recently shown that irradiation of aligned arrays of metallic nanowires with femtosecond laser pulses of relativistic intensity can volumetrically heat dense plasmas to multi-keV temperatures ${ }^{21}$, reaching pressures only achieved in the laboratory in spherical compression with the world largest lasers $^{22}$.

In the following, we demonstrate that the interaction of laser pulses of relativistic intensity with aligned deuterated nanostructures accelerates ions up to $\mathrm{MeV}$ energies in near-soliddensity media, opening a path to efficiently drive fusion reactions with joule-level lasers. We report a record in D-D neutron generation efficiency from plasmas generated by irradiating arrays of aligned high aspect ratio deuterated polyethylene $\left(\mathrm{CD}_{2}\right)$ with ultra-high contrast pulses of relativistic intensities from a compact laser.

\section{Results}

Arrays of aligned high aspect ratio nanowires have vacant spaces surrounding the wires (Fig. 1a) that allow for the deep penetration of ultrafast optical laser pulse energy into near-solid-density material, where light is trapped and practically totally absorbed ${ }^{21}$. Electrons are ripped off the nanowire surface by the large laser field and are accelerated to high energy in the voids. These energetic electrons interact with the nanowires, rapidly heating the material to extreme temperatures, causing the nanowires to explode (Fig. 1b-d). Ions are rapidly accelerated between the nanowires, and the voids are filled with plasma, creating a
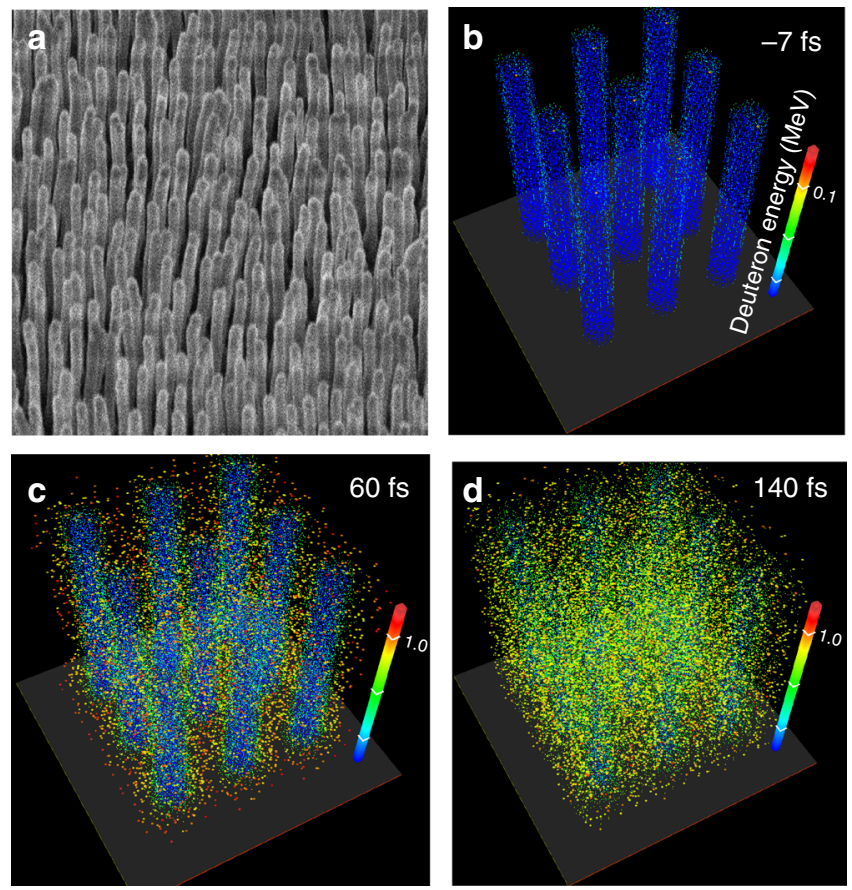

Fig. 1 SEM image and 3D PIC simulation of energy distribution of deuterons a SEM image of an array of $200 \mathrm{~nm}$ diameter $C D_{2}$ nanowires. b-d Threedimensional particle-in-cell (PIC) simulation of the evolution of the energy distribution of deuterons in an array of $400 \mathrm{~nm}$ diameter $C D_{2}$ nanowires irradiated at an intensity of $8 \times 10^{19} \mathrm{~W} \mathrm{~cm}^{-2}$ by an ultra-high contrast $\lambda=$ $400 \mathrm{~nm}$ laser pulse of $60 \mathrm{fs}$ FWHM duration. The laser pulses penetrate deep into the array where they rapidly heat the nanowires to extreme temperatures, causing the nanowires to explode (Fig. 1c, d). Deuterons are rapidly accelerated into the voids up to $M e V$ energies, producing D-D fusion reactions and characteristic $2.45 \mathrm{MeV}$ neutrons. Times are measured with respect to the peak of the laser pulse. The average density of the nanowire array corresponds to $16 \%$ solid density

continuous critical electron density layer that forbids further coupling of laser energy into the material (Fig. 1d). Assuming total laser energy absorption and volumetric heating of the target, the average energy per particle can be estimated to be:

$$
E_{\mathrm{av}} \approx \frac{a_{0}^{2}}{2} m c^{2} \frac{n_{\mathrm{c}}}{n_{\mathrm{av}}} \frac{Z}{(Z+1)} \frac{c \tau}{L},
$$

where $n_{\mathrm{c}}=\varepsilon_{0} m \omega_{0}^{2} / e^{2}$ is the critical electron density $\left(\mathrm{cm}^{-3}\right)$, with the average particle density $n_{\mathrm{av}}, \tau$ is the laser pulse duration, $Z$ is the mean ion charge, $L$ is the absorption depth in a target, and $m$ is the electron mass. The laser strength parameter, $a_{0}$, is defined as the normalized vector potential of the laser field and calculated as $a_{0}=0.855 \times 10^{-9} I^{1 / 2}\left(\mathrm{~W} \mathrm{~cm}^{-2}\right) \lambda_{0}(\mu \mathrm{m})$. For the conditions of the experiments discussed below $\left(a_{0} \sim 3, \lambda_{0}=400 \mathrm{~nm}, n_{\mathrm{av}}=6.4 \times\right.$ $10^{22} \mathrm{~cm}^{-3}, Z=2.67, \tau=60 \mathrm{fs}$, and $\left.L \sim 5 \mu \mathrm{m}\right), E_{\mathrm{av}}$ can be calculated to be $\sim 0.6 \mathrm{MeV}$. These energetic particles close the gaps between $400 \mathrm{~nm}$ diameter wires in an array with an average density corresponding to 15 percent solid density in $<100 \mathrm{fs}$. After homogenization of the material, the plasma as a whole, with ions of mass $M$, begins to expand in the normal direction toward the laser pulse with a characteristic time scale $\tau_{s} \approx \frac{L}{c} \sqrt{\frac{M c^{2}}{E_{\mathrm{av}}}} \sim 1.5 \mathrm{ps}$, but also toward the substrate, where the energetic deuterons moving into the target cause additional fusion reactions.

The use of sufficiently short laser pulses allows for very efficient coupling of the pulse energy deep into the nanowire array, heating to extreme temperatures a volume of near-solid-density material several microns in depth. This new approach to 
volumetric plasma heating opens access to the ultra-high energy density plasma regime using compact joule-class femtosecond lasers that can fire repetitively. We show below that the irradiation of deuterated nanowire arrays with pulses of relativistic intensity can accelerate a large number of deuterons to energies near the peak of the $\mathrm{D}-\mathrm{D}$ fusion cross-section, opening the possibility to efficiently drive $\mathrm{D}-\mathrm{D}$ fusion reactions and generate bright quasi-monoenergetic ultrashort neutron pulses from a point source with compact high repetition rate lasers. The particle-in-cell (PIC) simulation results illustrated in Fig. 1 show the computed spatio-temporal energy distribution of energetic deuterons in an array of $400 \mathrm{~nm}$ diameter deuterated polyethylene $\left(\mathrm{CD}_{2}\right)$ nanowires irradiated at an intensity of $8 \times 10^{19} \mathrm{~W}$ $\mathrm{cm}^{-2}$ by laser pulses of $60 \mathrm{fs}$ duration. The average density of the target was assumed to correspond to $16 \%$ of solid density. The plasma is rapidly fully ionized and the electron density is computed to reach $6.4 \times 10^{22} \mathrm{~cm}^{-3}$. The deuteron spectra calculated $60 \mathrm{fs}$ after the peak of the laser pulse shows energetic ions with kinetic energy up to $3 \mathrm{MeV}$ are generated. This energy greatly exceeds what we measured for D ions generated from solid targets irradiated under the same conditions. Moreover, the deep penetration of the heat in the nanowires results in a much larger volume of heated material, leading to the acceleration of a greater number of deuterons. In experiments conducted at these conditions, we observed a $\sim 500$ times increase in the number of 2.45 $\mathrm{MeV}$ D-D neutrons produced as compared to flat $\mathrm{CD}_{2}$ targets irradiated with the same laser pulses. The highest yield shots produced $2.2 \times 10^{6}$ neutrons per joule of laser energy. This corresponds to more than an order of magnitude increase with respect to the favorable yield reported for deuterated clusters ${ }^{9,15}$. We have also observed a strong superlinear increase of the neutron yield as a function of the laser irradiation intensity. The PIC simulations predict a further increase in irradiation intensity to $5 \times 10^{20} \mathrm{~W} \mathrm{~cm}^{-2}$ will shift the ion energy distribution to higher energies, practically depleting the population of the low energy deuterons and leading to a significant further increase in the neutron yield.

Experiments and Simulations. The experiments were conducted by irradiating arrays of aligned deuterated polyethylene $\left(\mathrm{CD}_{2}\right)$ nanowires with $\lambda=400 \mathrm{~nm}$, ultra-high contrast $\left(>10^{12}\right)$ pulses of $60 \mathrm{fs}$ FHWM duration with energy up to $1.65 \mathrm{~J}$. The experimental setup is schematically shown in Supplementary Figure 1. The laser pulses were generated by a frequency-doubled titanium: sapphire laser (Methods). The ultra-high contrast is necessary to prevent destruction of the nanowires prior to the arrival of the intense laser pulse. The laser pulses were focused at normal incidence onto the nanowire arrays using an $f / 1.7$ parabolic mirror. The energy spectra of the plasma ions were recorded using a Thomson parabola spectrometer (TPS, see Methods) placed at $75 \mathrm{~cm}$ from the target along the target normal. Ions reached the TPS through a $100 \mu \mathrm{m}$ hole on the axis of the focusing parabola. The primary diagnostics for neutron detection consisted of four EJ-228 plastic scintillator/photomultiplier detectors for time of flight measurements. In addition, two neutron bubble dosimeters were stationed outside the target chamber.

Measurements were conducted with arrays of aligned $\mathrm{CD}_{2}$ nanowires either 200 or $400 \mathrm{~nm}$ diameter and $\sim 5 \mu \mathrm{m}$ in length. The average density of the arrays corresponded to 16 and $19 \%$ solid density, respectively. We developed a method to fabricate the arrays of aligned $\mathrm{CD}_{2}$ nanowires by heated extrusion of $\mathrm{CD}_{2}$ into porous membranes (Methods). Flat solid targets of the same $\mathrm{CD}_{2}$ material and arrays of $\mathrm{CH}_{2}$ were also fabricated and shot for comparison with every $\mathrm{CD}_{2}$ nanowire array target. Fig. 2a shows a measured single-shot TPS spectrum from a flat $\mathrm{CD}_{2}$ target a

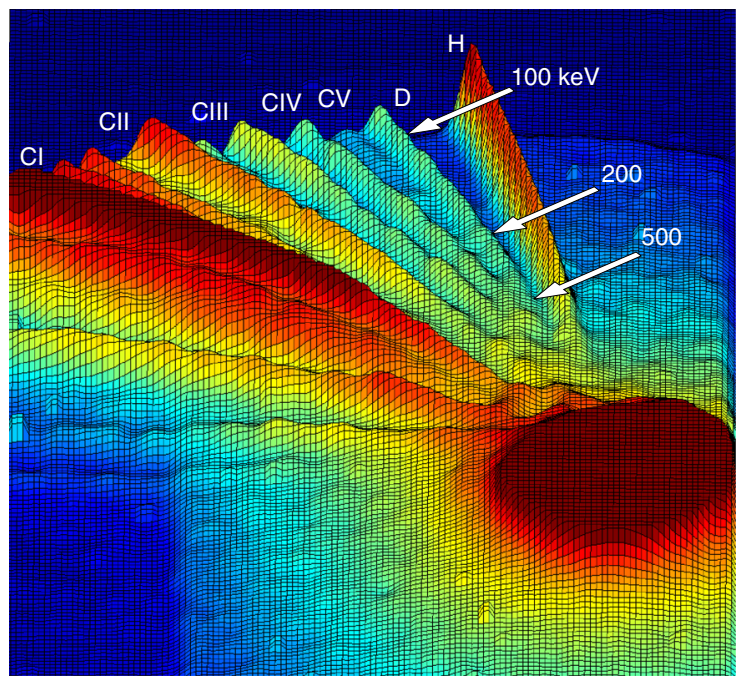

b
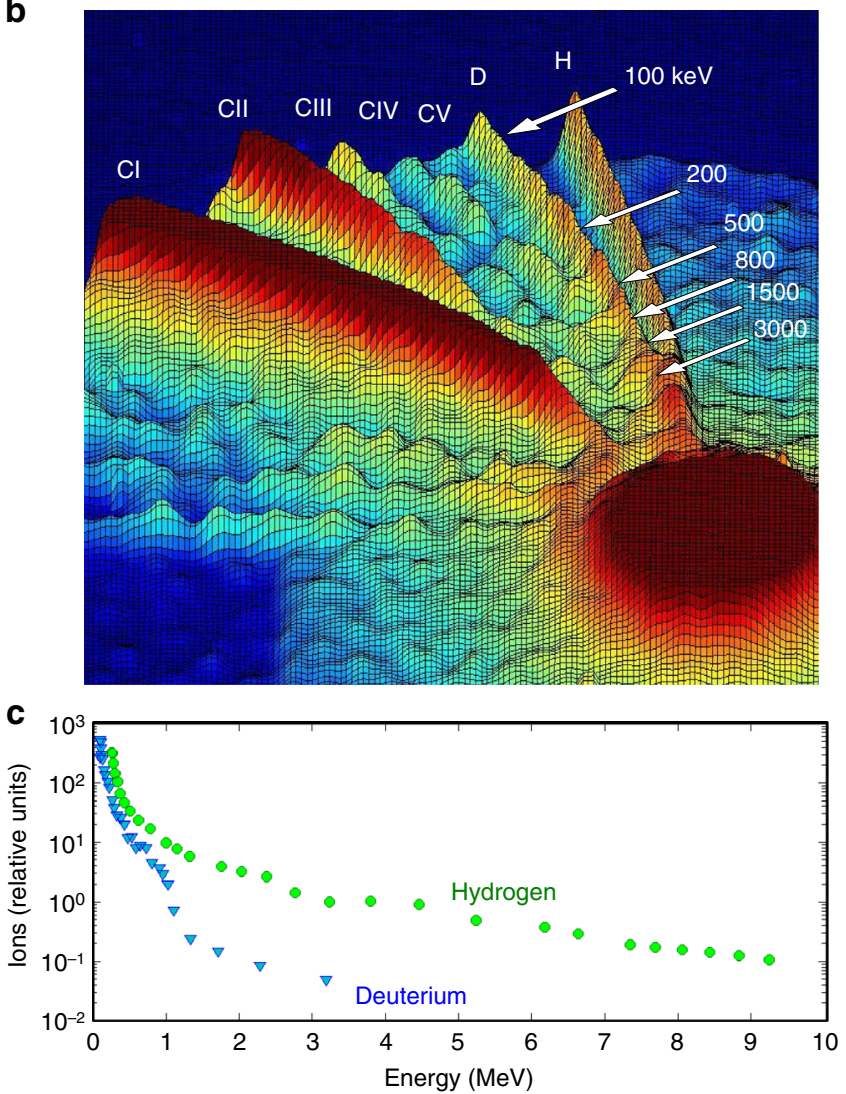

Fig. 2 Measured single-shot Thomson parabola ion energy spectra: a flat solid $\mathrm{CD}_{2}$ target irradiated at an intensity of $\sim 8 \times 10^{19} \mathrm{~W} \mathrm{~cm}^{-2}$. Traces corresponding to $\mathrm{H}, \mathrm{D}$, and $\mathrm{C}$ ions are recorded. The tail of the deuteron energy spectrum reaches $0.5 \mathrm{MeV}, \mathbf{b}$ array of $400 \mathrm{~nm}$ diameter, $5 \mu \mathrm{m}$ long, $C D_{2}$ nanowires. c Deuteron and proton energy distribution for the $C D_{2}$ nanowire array. The tail of the energy spectrum of the $D$ and $H$ spectra approaches $3 \mathrm{MeV}$ and $10 \mathrm{MeV}$, respectively

irradiated at an intensity of $8 \times 10^{19} \mathrm{~W} \mathrm{~cm}^{-2}$. Traces corresponding to $\mathrm{D}, \mathrm{H}$, and $\mathrm{C}$ ions are recorded. The energy of the deuterons in the flat solid target spectrum approaches $0.5 \mathrm{MeV}$. In comparison, arrays of $\mathrm{CD}_{2}$ nanowires irradiated at the same conditions are observed to produce deuterons of up to $3 \mathrm{MeV}$ energy and protons with energy as high as $10 \mathrm{MeV}$. A comparison of the ion energy spectra of $\mathrm{CD}_{2}$ and $\mathrm{CH}_{2}$ (Supplementary 
Figure 2) shows that the trace identified as corresponding to deuterons does not have a significant contribution of C VI ions, which have the same charge to mass ratio. The angular distribution of the fast deuterons was measured placing CR-39 plates at different angles from the target normal. To differentiate the deuteron flux from that of fast carbon ions, we covered part of each of the CR-39 plates with 2.5 or $4 \mu \mathrm{m}$ thick Al foils that stop practically all carbon ions and let through deuterons with energies $\gtrsim 270 \mathrm{keV}$ and $\gtrsim 400 \mathrm{keV}$, respectively. The deuteron flux was observed to peak in the direction of the target normal and to decrease as a function of angle, to become practically extinct for angles $>67$ degree (Supplementary Figure 3 ). Figure $2 c$ shows the deuteron and proton energy spectra extracted from Fig. $2 b$. The deuteron spectra is in good agreement with that resulting from the PIC simulations discussed below, and has a good overlap with the cross-section for D-D fusion reactions ${ }^{23}$. Consistently, time of flight traces recorded for several different target-detector distances show large neutron peaks at the expected arrival time for $2.45 \mathrm{MeV} \mathrm{D}-\mathrm{D}$ neutrons (Fig. 3 and Fig. 4). Data corresponding to solid $\mathrm{CD}_{2}$ flat targets is also shown in Fig. 4.

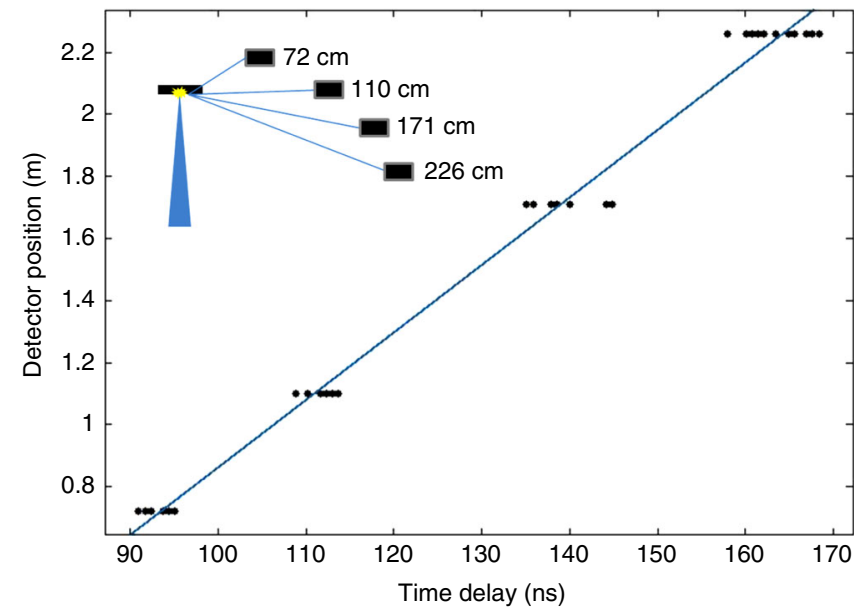

Fig. 3 Time of flight neutron measurements. Time of flight PMT/scintillator neutron data from four detectors located at different distances from the target. The trigger was the plasma $x$-ray signal preceding the neutrons. The slope of the line fitting the data corresponds to the velocity associated with $(2.48 \pm 0.14) \mathrm{MeV}$ neutrons

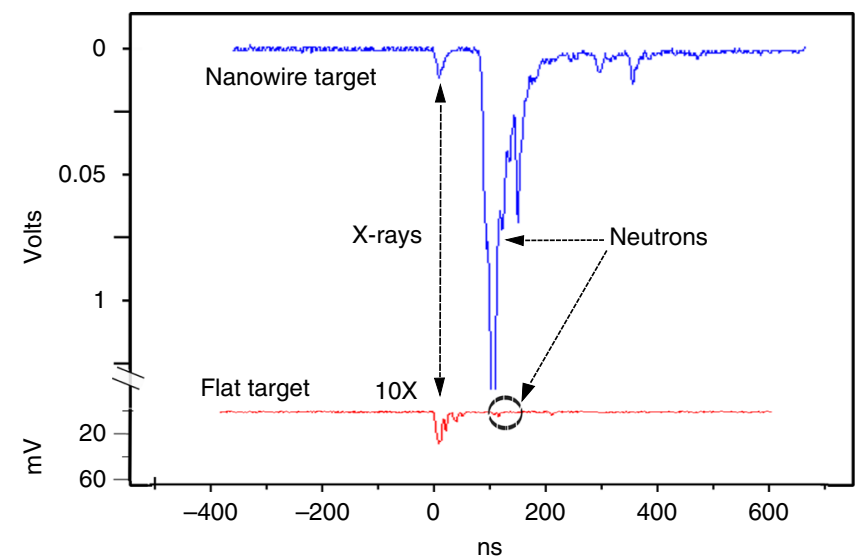

Fig. 4 Comparison of time of flight neutron signals. Comparison of TOF neutron signals from a nanowire array target and a flat target. The flat target neutron signal (in red) was multiplied by 10 for clarity. The ratio of the average neutron yield of eleven nanowire shots to the average yield of six flat target shots at the same irradiation conditions yielded a value of 492
When compared, the integrated neutron signals of the nanowire targets around $2.45 \mathrm{MeV}$ are $\sim 500$ times larger. The maximum number of neutrons per shot was measured to be $3.6 \times 10^{6}$ for a laser pulse energy of $1.64 \mathrm{~J}$, corresponding to $2.2 \times 10^{6}$ neutrons per joule, the largest fusion neutron yield obtained to date for joule-level laser pulse energies. If the same experiment with deuterated nanowires were to be conducted on a tritium containing substrate layer, the increase in the fusion crosssection combined with its shift towards lower ion energy, a significantly larger number of fusion neutrons could result.

Furthermore, the number of neutrons was measured to increase superlinearly with laser pulse energy (Fig. 5). The rapid increase is in good agreement with the simulations we conducted using the deuteron ion energy distributions resulting from the PIC simulations and nuclear kinetics. For these measurements and simulations, the laser spot size was kept constant while the pulse energy was varied. The neutron yield is a function of the D-D fusion cross-section and the stopping power for deuterons in the target material. The simulations show the measured increase in neutron yield is caused by a shift of the deuteron energy distribution to higher energies. This computed deuteron energy increase is illustrated in Fig. 6 for arrays of $\mathrm{CD}_{2}$ nanowires irradiated at intensities between $3 \times 10^{19} \mathrm{~W} \mathrm{~cm}^{-2}$ and $1 \times 10^{21} \mathrm{~W}$ $\mathrm{cm}^{-2}$. An increase of the intensity to $1 \times 10^{21} \mathrm{~W} \mathrm{~cm}^{-2}$ is seen to nearly deplete the low energy deuterons shifting a significant fraction of the distribution to multiple $\mathrm{MeV}$ energy. The simulations also show that at the lowest irradiation intensities used in this experiment, $\sim 1 \times 10^{19} \mathrm{~W} \mathrm{~cm}^{-2}$, the majority of the neutrons are produced within the plasma volume. In contrast, at the highest irradiation intensities explored, a significant fraction of the neutrons are produced in collision of accelerated deuterons with deuterium atoms in the material that surrounds the plasma. This includes neutrons generated by deuterons streaming into the $\sim 200 \mu \mathrm{m}$ thick $\mathrm{CD}_{2}$ substrate layer. This fraction increases with the irradiation intensity. At intensities $>1 \times 10^{21} \mathrm{~W} \mathrm{~cm}^{-2}$, the majority of the fusion reactions will occur in the substrate layer, outside the plasma volume. Therefore maximizing neutron

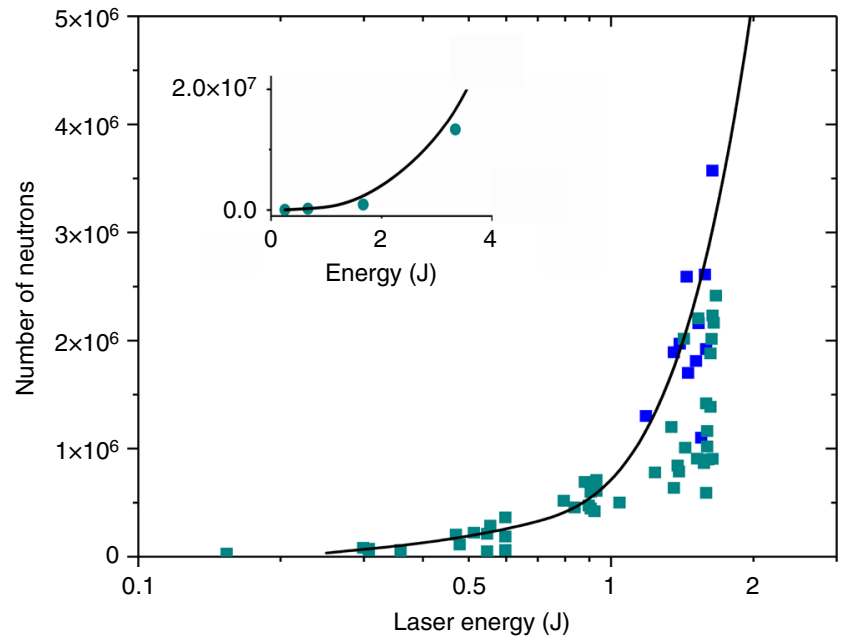

Fig. 5 Neutron yield as a function of laser pulse energy on target. The dark blue squares are shots corresponding to a target with $200 \mathrm{~nm}$ diameter wires. All the other shots (light blue squares) are for targets consisting of $400 \mathrm{~nm}$ diameter wire arrays. Each point resulted from the average of four scintillator/PMT time of flight detector signals. The line shows the simulated energy dependence of the neutron yield calculated using deuteron energy distributions computed by the PIC model and nuclear kinetics. The inset extends the simulation to $3.5 \mathrm{~J}$, where the green circles are computed values of the neutron yield 
production at higher irradiation intensities will require the use of a thicker $\mathrm{CD}_{2}$ substrate layer. Also, as the laser pulse energy is further increased beyond the values explored here, the optimum D-D neutron production might require a tradeoff between a further increase in the intensity and an increase in the irradiated volume. The higher intensities are also expected to generate a directed flux of high-energy deuterons that could be made to impinge in low $\mathrm{Z}$ convertors to drive "pitcher-catcher" neutron sources that have been demonstrated to create a large number of high-energy neutrons ${ }^{6,24,25}$. Finally, the simulations also show that the laser pulse drives a large forward electron current in the area around the wires. At higher irradiation intensities (eg. $5 \times$ $10^{21} \mathrm{~W} \mathrm{~cm}^{-2}$ ), this forward current is computed to induce return current densities of tens of Mega-amperes per $\mu \mathrm{m}^{2}$ through the nanowires ${ }^{26}$. The resulting strong quasi-static self-generated azimuthal magnetic field will pinch the deuterated nanowires into hot plasmas with a peak electron density exceeding 1000 times the critical density (Supplementary Figure 4).

In summary, we have realized a near-solid-density plasma regime in which deuterons from aligned nanostructures are accelerated up to $\mathrm{MeV}$ energies. The volumetric heating of aligned deuterated polyethylene nanowire arrays irradiated at relativistic intensity is shown to produce ultrashort neutron pulses with a $\sim 500$ times larger number of D-D neutrons than a deuterated flat solid target. A total of $2 \times 10^{6}$ neutrons per joule was generated, the largest $\mathrm{D}-\mathrm{D}$ fusion neutron yield reported to date for plasmas generated by laser pulse energies in the $1 \mathrm{~J}$ range. A further increase of the irradiation intensity is predicted to shift the deuteron energy distribution to significantly higher energies, which can be expected to lead into a further increase in D-D fusion reactions. This volumetrically heated dense fusion environment that can be created at a high repetition rates with compact lasers is of interest for high energy density science and nuclear science. The approach can also lead to the efficient generation of ultrafast pulses of quasi-monoenergetic neutrons from a point source for time-resolved material studies, ultrafast neutron radiography, and spectroscopy, and for high-energy science applications such as neutrino detector development.

\section{Methods}

Experimental Setup. Deuterated nanowire array targets were irradiated with ultrahigh contrast femtosecond laser pulses of up to $1.65 \mathrm{~J}$ from a frequency-doubled, $\lambda$ $=400 \mathrm{~nm}$, chirped-pulse-amplification titanium:sapphire laser. The pulses had 60 fs FWHM duration. The ultra-high contrast necessary to prevent destruction of the nanowires prior to the arrival of the main pulse was achieved by frequency doubling the compressed pulses in a $0.8 \mathrm{~mm}$ thick type $1 \mathrm{KDP}$ crystal. The conversion efficiency into second harmonic $(\lambda=400 \mathrm{~nm})$ was $\sim 40$ percent, which means the generation of the highest energy ultra-high contrast second harmonic pulses (1.64 J) required fundamental wavelength pulses of $\sim 6.5 \mathrm{~J}$ energy. The $400 \mathrm{~nm}$ wavelength second harmonic light was separated from the $800 \mathrm{~nm}$ fundamental beam using a sequence of four dichroic mirrors of 99.9 percent reflectivity at $400 \mathrm{~nm}$ (99.5 percent transmissive at the fundamental wavelength). The laser pulses were focused at normal incidence into a spot 2-2.6 $\mu \mathrm{m}$ diameter to achieve intensities up to $2 \times 10^{20} \mathrm{~W} \mathrm{~cm}^{-2}$ on target using an $\mathrm{f} / 1.7,90$ degree off-axis parabolic mirror. The focal spot size was determined by imaging with a $\times 50$ objective onto a 12-bit CMOS camera. Approximately 30 percent of the energy was concentrated in the central spot; the laser pulse duration was measured using single-shot frequency resolved optical gating. The laser pre-pulse contrast in the picosecond range was monitored with a third-order scanning autocorrelator, and in the nanosecond range, it was measured using the combination of a silicon photodiode and a set of calibrated neutral density filters. The intensity contrast of the frequency-doubled pulse is inferred to be $>1 \times 10^{12}$. The laser pulse energy on target was monitored on a shot-by-shot basis by measuring a calibrated leak through a mirror with $99 \%$ reflectivity at $\lambda=400 \mathrm{~nm}$, correcting for the reflectivity of the focusing parabola.

The ion energy distribution was measured using a TPS. The TPS built for these experiments uses two permanent Nd magnets, separated by a distance of $6 \mathrm{~mm}$, creating a field of 5000 Gauss. The magnets are biased with a potential difference of up to $2400 \mathrm{~V}$. A $6 \mathrm{~mm}$ diameter hole through the off-axis focusing parabola allows the ions to reach the TPS. The ions pass through a $100 \mu \mathrm{m}$ pinhole placed at $50 \mathrm{~cm}$ from the plasma and through the collinear fields to impinge on a pair of matched microchannel plates (MCPs) stacked in chevron configuration. The spatial information in the emitted electrons is transferred into a phosphor screen deposited onto an optical fiber bundle, which couples the fluorescence out of the vacuum to be imaged onto a charge coupled device (CCD) (Andor CCD). The TPS
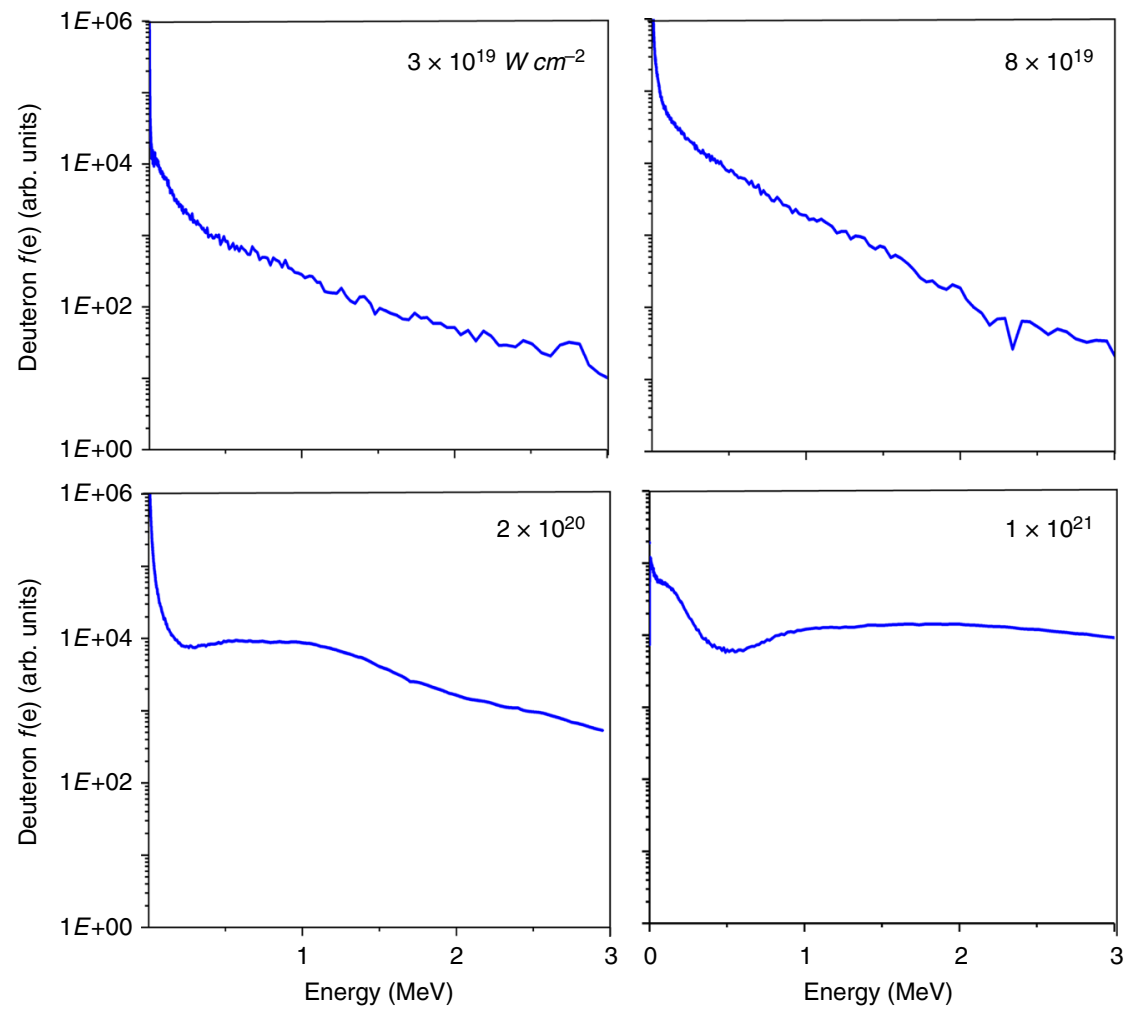

Fig. 6 Deuteron energy spectra ( $f(e)$, arb. units). Computed deuteron energy spectra for $400 \mathrm{~nm}$ diameter $C D_{2}$ nanowires at different irradiation intensities. The target average density corresponds to $16 \%$ of solid density 
was calibrated for ion energy by gating the $\mathrm{MCP}$ with respect to the time of arrival of the laser pulse to the target. The primary diagnostics for neutron detection consisted of four time of flight neutron detectors comprising EJ-228 plastic scintillators coupled to photomultiplier tubes (Hamamatsu H2431-50). The detectors were shielded by $10 \mathrm{~cm}$ of $\mathrm{Pb}$ on the side facing the plasma, and by $5 \mathrm{~cm}$ of $\mathrm{Pb}$ on all other sides in order to reduce the $\mathrm{X}$-ray signal reaching the photomultipliers. The neutron detectors were calibrated using the known neutron flux from a dense plasma focus at the National Security Technologies dense plasma focus facility ${ }^{27}$. The accuracy of the calibration is estimated to be $\sim 25$ percent, a value similar to the statistical fluctuations in the measurement of the number of neutrons. In addition, two neutron bubble dosimeters from Bubble Tech industries were stationed outside the chamber (with sensitivity 33 bubbles per mrem). The neutron number values inferred from the bubble counts corroborated the measurements from the scintillator detectors.

Deuterated Nanowire Arrays. The nanowire array targets were grown in house from deuterated polyethylene $\left(\mathrm{CD}_{2}\right)$ tested by infrared spectroscopy to contain $>99 \%$ deuterium vs hydrogen. Arrays of $200 \mathrm{~nm}$ and $400 \mathrm{~nm}$ diameter wires $\sim 5 \mu \mathrm{m}$ in length were used in the experiments, with an average density corresponding to 16 and $19 \%$ solid density, respectively. The wires were formed by heated extrusion into ion-tracked polycarbonate or alumina porous membranes for the arrays of 400 and $200 \mathrm{~nm}$ diameter wires, respectively. The nanowire arrays were exposed by dissolving the membranes. A substrate that contains a $\sim 200 \mu \mathrm{m}$ thick $\mathrm{CD}_{2}$ layer supports the nanowires. The morphology of each target array was characterized using scanning electron microscopy (SEM). The average target density can be determined by multiplying the density of the $\mathrm{CD}_{2}$ material used by the fraction of volume occupied by the nanowires. The latter is known from the porosity of the templates used, and was confirmed by SEM images of the nanowire arrays. The nanowire targets have a diameter of $12.5 \mathrm{~mm}$. To avoid shooting damaged regions, the target was displaced by $2 \mathrm{~mm}$ from the previous shot. This allowed us to acquire data from typically 12 shots per target. Flat $\mathrm{CD}_{2}$ targets were fabricated by heating the same material under pressure in a hydraulic press.

Simulation Tools. The PIC simulations were conducted using the relativistic threedimensional virtual laser-plasma laboratory (VLPL) $\operatorname{code}^{28}$. Its standard algorithms were extended by packages for optical-field ionization (OFI) and binary collisions, including electron impact ionization. OFI was treated as an under-barrier tunneling phenomenon in the static electric field ${ }^{29,30}$ with only sequential field ionization considered. The probabilities for Coulomb collisions between all particles in one mesh cell were calculated by a binary collision package. PIC simulations utilized a three-dimensional geometry and self-consistently included ionization physics. A linearly polarized plane wave with $400 \mathrm{~nm}$ wavelength and Gaussian time envelope $a(t)=a_{0} \exp \left(-t^{2} / \tau^{2}\right)$ was used to simulate the laser pulse where the normalized vector potential $a_{0}=3$ or 3.3 and a $60 \mathrm{fs}$ FWHM pulse duration. The laser pulse was assumed to impinge on the deuterated polyethylene nanowire array at normal incidence. The PIC simulation space consisted of a cell volume encompassing the wires and inter-wire gaps, as well as space above the array to allow for expansion of the wire material as it explodes and thermalizes. The grid size used ranged from $50 \times 50 \times 1120$ to $100 \times 100 \times 2208$ on a mesh volume of $0.81 \times 0.81 \times 6.2 \mu \mathrm{m}^{3}$. The time step was $0.00266 \mathrm{fs}$. The code accounts for local field enhancements, field fluctuations, and resonance heating. Simulations of neutron production dependence on laser irradiation energy were conducted with a post-processor code based on the code Radex ${ }^{31}$ using the ion energy distributions computed by the PIC code. The neutron yield is a function of the D-D fusion cross-section and the stopping power of a deuteron in the target material; both of these are energy-dependent. The stopping power was used to calculate the distance traveled by a particle in the material as it decreases in energy from $E_{\mathrm{i}}$ to $E_{\mathrm{i}+1}$ due to multiple scattering. Using the cross-section corresponding to this energy range, we calculate the probability of fusion in that interval. Integration of these values from the initial energy gives the total fusion probability for that deuteron. The cross sections were taken from the Evaluated Nuclear Data File database ${ }^{32}$. The stopping power was calculated using SRIM ${ }^{33}$. In the simulation shown in Fig. 5, the laser spot diameter was an adjustable parameter assumed to be $5 \mu \mathrm{m}$. Accurate neutron generation modeling at intensities significantly beyond $2 \mathrm{~J}$ will require a new model that takes into account effects, which can be neglected at energies of the experiments reported here but that will play a role at significantly higher intensities, such as heating of the substrate material.

Data Availability. All relevant data are available from the authors.

Received: 22 July 2017 Accepted: 14 February 2018

Published online: 14 March 2018

\section{References}

1. Dittrich, T. R. et al. Diagnosis of pusher-fuel mix in indirectly driven nova implosions. Phys. Rev. Lett. 73, 2324-2327 (1994).
2. Chang, P. Y. et al. Fusion yield enhancement in magnetized laser-driven implosions. Phys. Rev. Lett. 107, 35006 (2011).

3. Ren, G. et al. Neutron generation by laser-driven spherically convergent plasma fusion. Phys. Rev. Lett. 118, 165001 (2017).

4. Hurricane, O. A. et al. Fuel gain exceeding unity in an inertially confined fusion implosion. Nature 506, 343-348 (2014).

5. Klir, D. et al. Efficient neutron production from sub-nanosecond laser pulse accelerating deuterons on target front side. Phys. Plasmas 22, 93117 (2015).

6. Higginson, D. P. et al. Production of neutrons up to $18 \mathrm{MeV}$ in high-intensity, short-pulse laser matter interactions. Phys. Plasmas 18, 100703 (2011).

7. Key, M. H. et al. Hot electron production and heating by hot electrons in fast ignitor research. Phys. Plasmas 5, 1966-1972 (1998).

8. Kitagawa, Y. et al. Direct heating of a laser-imploded core by ultraintense laser-driven ions. Phys. Rev. Lett. 114, 195002 (2015).

9. Bang, W. et al. Optimum laser intensity for the production of energetic deuterium ions from laser-cluster interaction. Phys. Plasmas 20, 93104 (2013).

10. Strobl, M. et al. Advances in neutron radiography and tomography. J. Phys. $D$ Appl. Phys. 42, 243001 (2009).

11. Svergun, D. I. \& Koch, M. H. Advances in structure analysis using small-angle scattering in solution. Curr. Opin. Struct. Biol. 12, 654-660 (2002).

12. Prince, E. Neutron scattering instrumentation: a tutorial review. Appl. Spectrosc. Rev. 34, 159-172 (2004).

13. Pretzler, G. et al. Neutron production by $200 \mathrm{~mJ}$ ultrashort laser pulses. Phys. Rev. E 58, 1165-1168 (1998).

14. Norreys, P. A. et al. Neutron production from picosecond laser irradiation of deuterated targets at intensities of $1019 \mathrm{~W} \mathrm{~cm}^{-2}$. Plasma Phys. Control. Fusion 40, 175-182 (1998).

15. Ditmire, T., Zweiback, J., Yanovsky, V. P., Cowan, T. E. \& Hays, G. Nuclear fusion from explosions of femtosecond laser-heated deuterium clusters. Nature 398, 489-492 (1999).

16. Ditmire, T. et al. Nuclear fusion in gases of deuterium clusters heated with a femtosecond laser. Phys. Plasmas 7, 1993-1998 (2000).

17. Ditmire, T. et al. High-energy ions produced in explosions of superheated atomic clusters. Nature 386, 54-56 (1997).

18. Ditmire, T., Smith, R., Tisch, J. \& Hutchinson, M. High intensity laser absorption by gases of atomic clusters. Phys. Rev. Lett. 78, 3121-3124 (1997).

19. Grillon, G. et al. Deuterium-deuterium fusion dynamics in low-density molecular-cluster jets irradiated by intense ultrafast laser pulses. Phys. Rev. Lett. 89, 4-7 (2002).

20. Zweiback, J. et al. Detailed study of nuclear fusion from femtosecond laserdriven explosions of deuterium clusters. Phys. Plasmas 9, 3108 (2002).

21. Purvis, MA. et al. Relativistic plasma nanophotonics for ultrahigh energy density physics. Nat. Photonics 7, 796-800 (2013).

22. Glenzer, S. H. et al. Symmetric inertial confinement fusion implosions at ultrahigh laser energies. Science 327, 1228-1231 (2010).

23. Thompson, W. B. Thermonuclear reaction rates. Proc. Phys. Soc. Sect. B 70, 1-5 (1957).

24. Roth, M. et al. Bright laser-driven neutron source based on the relativistic transparency of solids. Phys. Rev. Lett. 110, 44802 (2013).

25. Zulick, C. et al. Energetic neutron beams generated from femtosecond laser plasma interactions. Appl. Phys. Lett. 102, 124101 (2013).

26. Kaymak, V., Pukhov, A., Shlyaptsev, V. N. \& Rocca, J. J. Nanoscale ultradense Z-pinch formation from laser-irradiated nanowire arrays. Phys. Rev. Lett. 117, 35004 (2016)

27. Goldin, F. J., Meehan, B. T., Hagen, E. C. \& Wilkins, P. R. Time-resolved spectra of dense plasma focus using spectrometer, streak camera, and CCD combination. Rev. Sci. Instrum. 81, 10E531 (2010).

28. Pukhov, A. \& Meyer-ter-Vehn, J. Laser hole boring into overdense plasma and relativistic electron currents for fast ignition of ICF targets. Phys. Rev. Lett. 79, 2686-2689 (1997).

29. Karmakar, A. \& Pukhov, A. Collimated attosecond $\mathrm{GeV}$ electron bunches from ionization of high- $Z$ material by radially polarized ultra-relativistic laser pulses. Laser Part. Beams 25, 371-377 (2007).

30. Zhidkov, A. \& Sasaki, A. Effect of field ionization on interaction of an intense subpicosecond laser pulse with foils. Phys. Plasmas 7, 1341-1344 (2000).

31. Shlyaptsev, V. N., Rocca, J. J. G., \& Osterheld, A. L. Dynamics of a capillary discharge x-ray laser. in Proceedings of the SPIE (eds. Rocca, J. J. G. \& Hagelstein, P. L.) Vol. 2520, 365-372 (International Society for Optics and Photonics, 1995).

32. IAEA-NDS. ENDF: Evaluated Nuclear Data File. (2017). Available at: https:// www-nds.iaea.org/exfor/endf.htm.

33. Ziegler, J. James Ziegler - SRIM \& TRIM. (2017) http://www.srim.org/.

\section{Acknowledgements}

This material is based on work supported by the Air Force Office of Scientific Research under award number FA9560-14-10232 and FA9550-17-1-0278, and by Mission Support and Test Services, LLC. The simulations were conducted using the CU-CSU Sumit Computer Facility, NSF (ACI-1532235). We acknowledge the contributions of Zhenlin 
Su and Jose Moreno in the development of the Thomson parabola and Conrad Buss for fabricating the nanowire targets.

\section{Author Contributions}

J.J.R. and V.N.S. conceived the experiment. A.P. developed the PIC code. V.K. and A.P. preformed simulations and V.N.S. provided theoretical support. A.C., C.C., J.T., R.H., Y. W, S.W., and A.R. conducted the experiment, C.C. and A.C. fabricated the targets, J.T. calibrated the neutron detectors, Y.W., S.W., A.R., and J.J.R. developed the laser, and R. H., A.C., C.C., and J.J.R. developed the high contrast beamline. All the authors contributed to writing the paper.

\section{Additional information}

Supplementary Information accompanies this paper at https://doi.org/10.1038/s41467018-03445-z.

Competing Interests: The authors declare no competing interests.

Reprints and permission information is available online at http://npg.nature.com/ reprintsandpermissions/
Publisher's note: Springer Nature remains neutral with regard to jurisdictional claims in published maps and institutional affiliations.

(c) (i) Open Access This article is licensed under a Creative Commons Attribution 4.0 International License, which permits use, sharing, adaptation, distribution and reproduction in any medium or format, as long as you give appropriate credit to the original author(s) and the source, provide a link to the Creative Commons license, and indicate if changes were made. The images or other third party material in this article are included in the article's Creative Commons license, unless indicated otherwise in a credit line to the material. If material is not included in the article's Creative Commons license and your intended use is not permitted by statutory regulation or exceeds the permitted use, you will need to obtain permission directly from the copyright holder. To view a copy of this license, visit http://creativecommons.org/ licenses/by/4.0/.

(C) The Author(s) 2018 